\title{
Genetic Diversity of Plasmodium falciparum Strains in Children under Five Years of Age in Southeastern Tanzania
}

\author{
Deborah Sumari $^{*}, 1$ Ken M. Hosea ${ }^{2}$, Joseph P. Mugasa ${ }^{1,3}$ and Salim Abdulla ${ }^{1}$ \\ ${ }^{I}$ Ifakara Health Institute, Tanzania, ${ }^{2}$ Department of Molecular Biology and Biotechnology, University of Dar es Salaam, \\ Tanzania, ${ }^{3}$ Glasgow Biomedical Research Centre, University of Glasgow, Scotland, UK
}

\begin{abstract}
Strain diversity may play a role in delaying development of protective immunity in endemic areas. We evaluated genetic diversity of Plasmodium falciparum infected children before being treated with Sulphadoxine Pyrimethamine (SP) and Coartem ${ }^{\mathrm{TM}}$ in Southeastern Tanzania. Allelic diversity of $P$. falciparum strains were determined in order to further assist in correct estimation of recrudescent and new infections.

P. falciparum isolated from 300 children aged 1-59 months was used in the study, where nested PCR followed by Restriction Fragment Length Polymorphism (RFLP) of highly polymorphic Merozoite surface protein 2 ( $m s p 2$ ) was employed to understand the genetic diversity of the parasites population. Frequency of $m s p 2$ gene alleles was calculated and further associated with multiplicity of infection of children under five years of age.

A total of 71 and 83 different msp 2 alleles were found in Rufiji and Ulanga districts respectively. Children infected with either FC27 or 3D7 allelic type in Rufiji were 42\% single, 55.3\% double and 2.7\% triple, while in Ulanga, 36.7\% single, $62 \%$ double and $1.3 \%$ triple infections. Mean numbers of multiplicity of infections (MOI) in Rufiji and Ulanga were 1.6 and 1.3, respectively. These findings show a high genetic diversity of $P$. falciparum strains in study areas and low MOI could reflect production of susceptible parasites that immune response can accommodate or can be cleared by the drugs. Furthermore, 3D7 allelic type of msp2 gene was more prevalent than FC27 in Ulanga district, indicating association between $m s p 2$ allelic type and disease severity, hence predict possible vaccine candidate in the future.
\end{abstract}

Keywords: P. falciparum, genetic diversity, $m s p 2$ gene, Tanzania.

\section{INTRODUCTION}

High malaria transmission in many parts of the world has been associated with high Plasmodium falciparum genetic diversity $[1,2]$. Genetic diversity as a result of genetic recombination increases the risk of an individual to be infected with different parasite genotypes even if the area has stable malaria transmission. Such infections are generally described as complex or multiple infections [2] and have previously been explored on grounds of genetic variation and its effects on parasite population dynamics [3].

Understanding the patterns and mechanisms of DNA sequence variation in major $P$. falciparum surface antigens is critical for the purpose of predicting effectiveness of human host immune response [4]. Moreover, various studies have shown that most endemic areas inhabited by $P$. falciparum are infected with mixtures of genetically distinct clones [5]. It has also been reported that high polymorphism featured in certain surface proteins such as Merozoite surface proteins (MSP) of $P$. falciparum has even hindered vaccine development [6].

Merozoite surface protein 1 (MSP1), Merozoite surface protein 2 (MSP2) and glutamate-rich protein (GLURP) are the most commonly used markers for identifying $P$. falciparum populations diversity. The $m s p 2$ gene has been

*Address correspondence to this author at the Ifakara Health Institute, P.O. Box 78373, Tanzania; Tel: +255222774714; Fax: +255222771714;

E-mail: dsumari@ihi.or.tz extensively studied and is so far the most informative single diversity marker [7]. Its polymorphic features have been exploited for studying naturally occurring parasite populations [7, 8]. The $m s p 2$ alleles generally fall into two allelic families, FC27 and 3D7, which differ considerably by the dimorphic structure of the variable central region [9]. Studies conducted in Central African Republic and other areas suggested that, the epidemiological features of $P$. falciparum surface proteins may vary significantly within areas $[10,11]$. Furthermore, several studies have associated parasite diversity with treatment outcome [12] but this might not be the case in endemic areas with stable transmission where the immune system can play a major role in parasite clearance or reduction of severity $[13,14]$.

Here we report the genetic diversity of $P$. falciparum strains among children and apparent number of concurrent infections per blood sample of an individual (MOI) before treatment with Sulfadoxine-Pyrimethamine (SP) and Artemisinin-based Combination Therapy. We enrolled children aged less than five years from Ulanga and Rufiji districts, where as molecular techniques were used so as to provide evidence on genetic diversity status of the parasite strains in these areas and particularly the study population.

\section{MATERIALS AND METHODS}

The study was carried out in Southeastern Tanzania in Kibiti, Rufiji district and Lupiro in Ulanga district. It was a cross-sectional study conducted in 2004 as part of the big 
project that aimed to assess combination therapy among children with uncomplicated malaria. Rufiji is a rural district located $178 \mathrm{~km}$ south of Dar es Salaam along the Indian Ocean coast with an area of $14,500 \mathrm{~km}^{2}$ and a population of 203,102 . Just like the former, Ulanga is also a rural district but this is found, along the Kilombero river valley about 500 $\mathrm{km}$ Southeast of Dar es Salaam with a population of 194,209 [15]. Malaria transmission in Rufiji and Ulanga districts is intense and perennial. The catchment populations at the two health centers in Kibiti-Rufiji and Lupiro-Ulanga were 26,822 and 40,385 respectively.

\section{Ethical Issues}

The study obtained ethical clearance from both Institution Review Board (IRB) and national Institute for Medical research (NIMR) ethical committee authorities with the project license No.1810. Oral and written informed consent about risks and benefits that may occur were presented to the parents or guardians of the participating children. The parents and guardians accepted and gave informed consent for the participation in the study.

\section{Sample Collection}

We enrolled children aged less than five years from Ulanga and Rufiji districts and randomly selected a total of 300 children with uncomplicated malaria before treatment from the two districts. A total of 150 samples were collected from each district, Ulanga and Rufiji. Thereafter, blood samples were spotted on filter papers (3MM Whatman) in a sterile manner, dried and properly stored at room temperature until DNA isolation and molecular analysis was done. Polymerase Chain Reaction (PCR) as a molecular technique was performed followed by restriction fragment length analysis (PCR-RFLP) of msp2 genes on DNA isolates. Blood samples were collected before drug administration according to WHO in vivo standard protocol [16].

\section{DNA Extraction and PCR-RFLP}

$P$. falciparum genomic DNA was extracted from blood collected on filter paper by modified Chelex method as previously described [17]. The extracted DNA from each sample was used immediately for PCR and the remaining portion was stored at $-20^{\circ} \mathrm{C}$ in appropriately labeled storage tubes. Nested PCR followed by RFLP analysis was carried out to amplify $m s p 2$ gene using primer sets and restriction enzyme for allelic families analysis as previously detailed by Felger et al. 1999 [18]. Restricted PCR fragments were sized using DNA marker (Solis BioDyne) as a reference. The reference DNA marker is also known as DNA ladder as its fragment sizes increase from the bottom and hence makes sizing of the PCR fragments easy.

\section{Statistical Analysis}

Data were analyzed using Chi-square test and Shannonweaver index of diversity. Chi-square test was used to compare the allelic families of $m s p 2$ gene in the two districts [18]. MOI was performed using Shannon-weaver index of diversity as detailed in Hutcheson, 1970 [19] but with some modifications.

\section{RESULTS}

All 300 blood samples from the study population were successfully analyzed for parasite genetic diversity and MOI. In Rufiji, 40\% (60/150) were FC27, 43\% (65/150) 3D7 and $17 \%(25 / 150)$ mixed infections, whereas in Ulanga $36.7 \%$ (55/150) were FC27, 56\% (84/150) 3D7 and 7.3\% (11/150) mixed infections. There was a significant difference in distribution of 3D7 and FC27 in Ulanga district $(P<0.05)$ but not in Rufiji $(P>0.05)$.

MOI in children from Rufiji and Ulanga were as follows; Children with single infections of either FC27 or 3D7 allelic type in Rufiji were $42 \%(63 / 150)$, those with double infections were $55.3 \%(83 / 150)$, and $2.7 \%(4 / 150)$ had triple infections. In Ulanga, 36.7\% (55/150) single, 62\% (93/150) double and $1.3 \%(2 / 150)$ triple infections (Fig. 1). The mean numbers of MOI were 1.6 and 1.3 in Rufiji and Ulanga, respectively, with a range of 1-3 genotypes per individual.

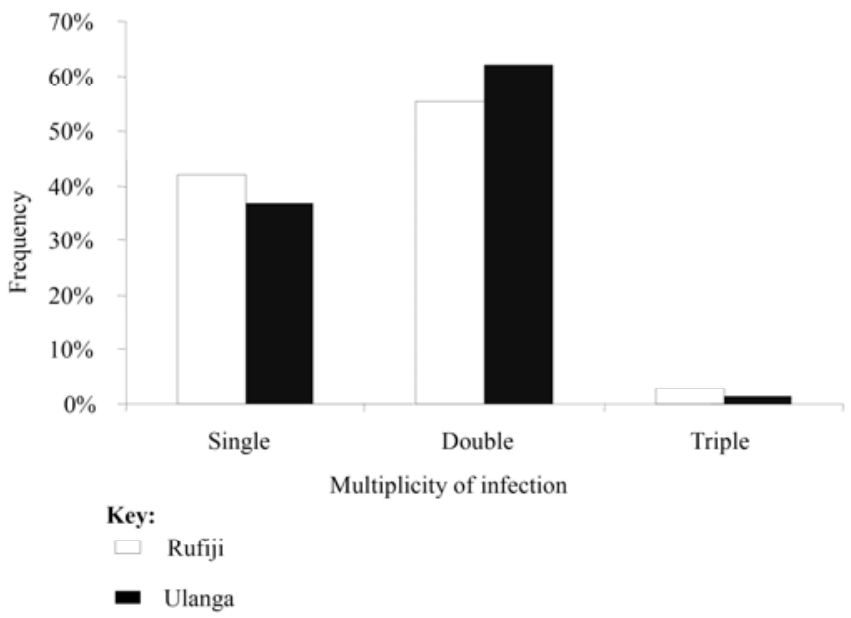

Fig. (1). Frequency distribution of concurrent infections among children under five years of age in Rufiji and Ulanga districts.

Out of 150 infected children from Rufiji, 71 different msp2 alleles were detected, 36 alleles belonged to 3D7 and 35 belonged to FC27 with no statistical difference between the two families ( $\mathrm{df}=70, P>0.05$ ). On the other hand, out of the 83 different $m s p 2$ alleles detected in Ulanga, 45 alleles belonged to 3D7 and 38 belonged to FC27 with clear significant difference between the two families ( df $=83, P>$ 0.05). A total of 69 children both in Ulanga and Rufiji districts were infected with parasites containing 3D7 and FC27 alleles having base pairs of 210, 270, 300, 350 and 400 (Tables 1 and 2). This indicates that, amplicons of the same size can be found in the two allelic families (3D7 and FC27). This may lead to difficulties in distinguishing recrudescence and new infection basing on these amplicons.

\section{DISCUSSION}

In this study we found 71 and 83 different msp 2 alleles in study samples collected from Rufiji and Ulanga respectively, with 3D7 allelic types featuring the most followed by FC27 in both districts. Genetic diversity of $P$. falciparum strains in the study population was found to be high in both districts which indicate that close ecologically related areas would have similar distribution of parasite strains. This may have an implication on treatment of malaria among children living 
Table 1. The Allelic Diversity of $P$. falciparum msp2 Allelic Families Occurred in Rufiji

\begin{tabular}{|c|c|c|c|c|c|c|c|}
\hline Alleles of 3D7(bp) & Frequency (fi) & fi $\log f i$ & fi $\log 2 \mathrm{fi}$ & Alleles of FC27(bp) & Frequency (fi) & fi $\log f i$ & fi $\log 2 \mathrm{fi}$ \\
\hline 3D7200 & 3 & 1.4314 & 0.6829 & FC27210 & 6 & 3.4949 & 2.4428 \\
\hline 3D7400 & 4 & 2.4082 & 1.4499 & FC27300 & 3 & 1.4314 & 0.6829 \\
\hline 3D7300 & 5 & 3.4949 & 2.4428 & FC27430 & 2 & 0.6021 & 0.1812 \\
\hline 3D7210 & 4 & 2.4082 & 1.4499 & FC27270 & 4 & 2.4082 & 1.4499 \\
\hline 3D7360 & 4 & 2.4082 & 1.4499 & FC27450 & 3 & 1.4314 & 0.6829 \\
\hline 3D7270 & 3 & 1.4314 & 0.6829 & FC27120 & 3 & 1.4314 & 0.6829 \\
\hline 3D7450 & 4 & 2.4082 & 1.4499 & FC27220 & 4 & 3.4949 & 2.4428 \\
\hline
\end{tabular}

Table 2. The Allelic Diversity of P. falciparum msp2 Allelic Families Occurred in Ulanga

\begin{tabular}{|c|c|c|c|c|c|c|c|}
\hline 3D7270 & 5 & 3.4949 & 2.4428 & FC27120 & 3 & 1.4314 & 0.6829 \\
\hline 3D7370 & 4 & 2.4082 & 1.4499 & FC27250 & 3 & 1.4314 & 0.6829 \\
\hline 3D7320 & 2 & 0.6021 & 0.1812 & FC27210 & 5 & 3.4949 & 2.4428 \\
\hline 3D7210 & 2 & 0.6021 & 0.1812 & FC27260 & 2 & 0.6021 & 0.1812 \\
\hline 3D7360 & 3 & 1.4314 & 0.6829 & FC27245 & 2 & 0.6021 & 0.1812 \\
\hline 3D7300 & 4 & 2.4082 & 1.4499 & FC27510 & 1 & 0 & 0 \\
\hline 3D7400 & 6 & 4.6689 & 3.6331 & FC27270 & 5 & 3.4949 & 2.4428 \\
\hline Total & $\mathrm{N}=45$ & 27.2445 & 17.6439 & & $\mathrm{n}=38$ & 20.1674 & 11.5623 \\
\hline
\end{tabular}

in endemic areas where immunity is stable and drug resistance issues are to be considered. These findings corroborate with those from Dakar, Senegal [20] and Mlimba, Tanzania [21], which reported 3D7 alleles to be more prevalent than FC27 in those infected with single, double and triple infections. Nevertheless, different proportions of the two allelic families in Ulanga observed in this study corroborate with the findings obtained in Dielmo, Senegal [22, 23] and Sao Tome [24]. This difference of the two allelic families has been proposed to arise due to the presence of considerable heterogeneity in parasite populations [23]. On the other hand, results obtained from Rufiji district in this study indicate that, there is no significant difference in the prevalence of the two families. This is similar to findings reported by previous studies done in Kilombero, Tanzania [18], Ndiop, Senegal [25] and Papua New Guinea [26].
The diversity of $m s p 2$ alleles in Rufiji observed between the families in this study supports suggestion by Felger et al. 1999 [18] that, although the genetic diversity is extensive, it is limited by structural constraints or immune selection. In this study we found $63.3 \%$ and $58 \%$ of the individuals harbor more than one infection in Ulanga and Rufiji respectively, as compared to only $25 \%$ obtained in Papua New Guinea [23]. The high prevalence of multiple $P$. falciparum infections being reported by this study is broadly consistent with previous findings from other parts of Tanzania including Michenga village [27].

Furthermore, results from this study show that establishment of the specific DNA fragments is a useful indicator for evaluating genetic diversity of parasite populations. It is important to note that, there are similar PCR amplicon sizes in the two families of which can lead to wrong results if their diversity has not been clearly defined. It will then be a 
challenge to distinguish recrudescence from re-infection in settings where amplicons are not sequenced to remove possible discrepancies.

Furthermore in endemic areas, the number of different clones of malaria parasites co-infecting a single host (MOI) can be a useful indicator of the level of transmission and/or the immune status of the host [28]. Increase in transmission levels (Entomological Inoculation Rates) is generally associated with progressive increases in the average number of parasite clones per host [27-29].

Mean MOIs obtained in this study were lower (1.6 and 1.3 in Rufiji and Ulanga, respectively) than those obtained in other areas in Tanzania such as, Kilombero, Mkuranga, Mlimba and Kyela [18, 30]. Felger et al. 1999 [18] reported the average MOI of 4 in symptomatic children in Kilombero, while Mugittu et al. 2005 [30] recorded 2.5 in asymptomatic children in Mkuranga, Mlimba and Kyela. Studies done by Beck et al. 1997 [31] reported a MOI of 5 in Senegal. The reason for this observation could be due to technical problems such as lower detection limits of some clones by PCR at low densities, it can as well be speculated due to difference in bed net usage, difference in transmission and treatment seeking behavior of individuals living in the two areas. In previous studies elsewhere in the world, it has also been demonstrated that multiplicity of infection is age dependent but may differ in areas with malaria transmission [22, 32, 33]. However, this factor was not evaluated in this study since samples and data available were only from children less than five years of age. Generally, our findings stress on the presence of highly diverse $P$. falciparum infections in these areas before treatment and created useful information on the presence of the same DNA fragments in both allelic families of the msp 2 gene.

\section{CONCLUSION}

In this study we found a high number of $P$. falciparum strains in symptomatic children in Ulanga and Rufiji districts. Most of these strains harbor double infections although the frequency distribution of the two allelic families varied slightly between the two districts. The high parasite diversity indeed indicates high transmission levels in these areas. We anticipate our findings to provide a baseline to detect and describe the parasitological outcome of vaccine development trials and perhaps elucidate biological effects of the vaccine candidate for malaria. Moreover, our findings will help future studies explore allelic diversity of the parasite strains to correctly distinguish recrudescence from new infection in areas where malaria transmission is intense throughout the year.

\section{AUTHORS' CONTRIBUTION}

DS drafted the manuscript, designed the experiment, coordinated data collection and analyzed data. $\mathrm{KMH}$ and JPM participated to design the study and manuscript development. SA supervised the work from inception, acquisition of funding, and development of the study protocol on the earlier version. All authors read and approved the final version of the manuscript.

\section{ACKNOWLEDGEMENTS}

This work was funded by CDC, Atlanta under IMPACT$\mathrm{Tz}$ project through CDC Cooperative agreement number UR3/CCU018969 and all laboratory work was carried out in Ifakara Health Institute Laboratory. We are grateful to all field workers and all parents and guardians who consented for their children to participate in the study. We also appreciate contribution shown by Ifakara Lab technicians on collection of blood filter papers and reading of blood slides. Special thanks go to Dr. Wagner of the University of Dar es Salaam for his support in the choice of proper statistical package to be used for analyzing data in this study.

\section{REFERENCES}

[1] Contamin H, Fandeur T, Bonnefoy S, Skouri F, Ntoumi F, Mercereau-Puijalen O. PCR typing of field isolates of Plasmodium falciparum. J Clin Microbiol 1995; 33: 944-951.

[2] Babiker HA, Lines J, Hill WG, Walliker D. Population structure of Plasmodium falciparum in villages with different malaria endemicity in East Africa. Am J Trop Med Hyg 1997; 56: 141-147.

[3] Paul RE, Day KP. Mating patterns of Plasmodium falciparum. Parasitol Today 1998; 14: 197-202.

[4] Hartl DL, Volkman SK, Nielsen KM, et al. The paradoxical population structure of Plasmodium falciparum. Trend Parasitol 2002; 18: 266-72.

[5] Magesa SM, Mdira KY, Babiker HA, et al. Diversity of Plasmodium falciparum clones infecting children in a holoendemic area in north-eastern Tanzania. Acta Trop 2002; 84: 83-92.

[6] Hughes AL, Verra F. Extensive polymorphism and ancient origin of Plasmodium falciparum. Trends Parasitol 2002; 18: 348-351.

[7] Farnert A, Arez AP, Babiker HA, et al. Genotyping of Plasmodium falciparum infections by PCR: a comparative multicentre study. Trans R. Soc Trop Med Hyg 2001; 95: 225-32.

[8] Basco LK, Tahar R, Escalante A. Molecular epidemiology of malaria in Cameroon. XVIII. Polymorphisms of the Plasmodium falciparum Merozoite surface antigen-2 gene in isolates from symptomatic patients. Am J Trop Med Hyg 2004; 70: 238-44.

[9] Felger I, Steiger S, Hatz C, Smith T, Beck HP. Antigenic crossreactivity between different alleles of the Plasmodium falciparum merozoite surface protein 2. Parasite Immunol 2003; 25: 531-43.

[10] Nicastri E, Paglia MG, Severini C, Ghirga P, Bevilacqua N. Plasmodium falciparum multiple infections, disease severity and host characteristics in malaria affected travelers returning from Africa. Travel Med Infect Dis 2008; 6: 205-9.

[11] Dolmazon V, Matsika-Claquin MD, Manirakiza A, et al. Genetic diversity and genotype multiplicity of Plasmodium falciparum infections in symptomatic individuals living in Bangui (CAR). Acta Trop 2008; 107: 37-42.

[12] Basco L, Ringwald P. Molecular epidemiology of malaria in Younde, Cameroon. VIII. Multiple Plasmodium falciparum infections in symptomatic patients. Am J Trop Med Hyg 2001; 65: 798-803.

[13] Robert V, Roeffen W, Brasseur P, et al. Anti-NANP antibody and treatment efficacy in patients with acute uncomplicated falciparum malaria attacks. Parasite Immunol 2000; 22: 589-93.

[14] Mayxay M, Chotivanich K, Pukrittayakamee S, et al. Contribution of humoral immunity to the therapeutic response in falciparum malaria. Am J Trop Med Hyg 2001; 65: 918-23.

[15] Tanzania national census. Population and housing census general report, 2002. [Cited July, 2005]. Available at: http://www.tanzania.go.tz/census/districts.htm

[16] World Health Organization. The use of antimalarial drugs. Report of an informal consultation. 13-17 November 2001, Geneva: WHO 2002.

[17] Plowe CV, Djimde A, Bouare M, Doumbo O, Wellems TE. Pyrimethamine and proguanil resistance-conferring mutations in Plasmodium falciparum dihydrofolate reductase: polymerase chain reaction methods for surveillance in Africa. Am J Trop Med Hyg 1995; 52: 565-8.

[18] Felger I, Irion A, Steiger S, Beck HP. Epidemiology of multiple Plasmodium falciparum infections 2. Genotypes of merozoite 
surface protein 2 of Plasmodium falciparum in Tanzania. Trans R Soc Trop Med Hyg 1999; 93: 3-9.

[19] Hutcheson K. A test for comparing diversities based on Shannon formula. J Theor Biol 1970; 29: 151-4.

[20] Henry M, Diallo I, Bordes J, et al. Urban malaria in Dakar, Senegal: Chemosusceptibility and genetic diversity of Plasmodium falciparum isolates. Am J Trop Med Hyg 2006; 75: 146-51.

[21] Mbugi EV, Mutayoba BM, Balthazary ST, et al. Multiplicity of infections and level of recrudescence in Plasmodium falciparum malaria in Mlimba, Tanzania. Afr J Biotechnol 2006; 5: 1655-62.

[22] Ntoumi F, Contamin H, Rogier C, et al. Age-dependent carriage of multiple Plasmodium falciparum merozoite surface antigen-2 alleles in asymptomatic malaria infections. Am J Trop Med Hyg 1995; 52: 81-88.

[23] Ntoumi F, Mercereau-Puijalon O, Ossari S, et al. Plasmodium falciparum: sickle-cell trait is associated with higher prevalence of multiple infections in Gabonese children with asymptomatic infections. Exp Parasitol 1997; 87: 39-46.

[24] Muller DA, Charlwood JD, Felger I, Ferreira C, Smith T. Prospective risk of morbidity in relation to multiplicity of infection with Plasmodium falciparum, Sao Tome. Acta 2001; 78: 155-62.

[25] Zwetyenga J, Rogier C, Tall A, et al. No influence of age on infection complexity and allelic distribution in Plasmodium falciparum infections in Ndiop, a Senegalese village with seasonal, mesoendemic malaria. Am J Trop Med Hyg 1998; 59: 726-35.

[26] Felger I, Tavul L, Kabintik S, et al. Plasmodium falciparum: extensive polymorphism in merozoite surface antigen 2 alleles in an area with endemic malaria in Papua New Guinea. Exp Parasitol 1994; 79: 106-16.
Babiker HA, Ranford-Cartwright LC, Walliker D. Epidemiology of multiple Plasmodium falciparum infections. 3. Genetic structure and dynamics of Plasmodium falciparum infections in the Kilombero region of Tanzania. Trans R Soc Trop Med Hyg 1999; 93: S1/11-S1/14.

[28] Owusu-Agyei S, Smith T, Beck HP, Amenga-Etego L, Felger I. Molecular epidemiology of Plasmodium falciparum infections among asymptomatic inhabitants of a holoendemic malarious area in northern Ghana. Trop Med Int Health 2002; 7: 421.

[29] Arnot D. Unstable malaria in Sudan: the influence of the dry season. Clone multiplicity of Plasmodium falciparum infections in individuals exposed to variable levels of disease transmission. Trans R Soc Trop Med Hyg 1998; 92: 580-5.

[30] Mugittu K, Abdulla S, Falk N, et al. B: Efficacy of sulfadoxinepyrimethamine in Tanzania after two years as first-line drug for uncomplicated malaria: assessment protocol and implication for treatment policy strategies. Malar J 2005; 4: 55 .

[31] Beck HP, Felger I, Huber W, et al. Analysis of multiple Plasmodium falciparum infections in Tanzanian children during the phase III trial of the malaria vaccine SPf66. J Infect Dis 1997; 175: 921-6.

[32] Smith T, Beck HP, Kitua A, et al. Epidemiology of multiple Plasmodium falciparum infections. 3. Age dependence of the multiplicity of Plasmodium falciparum infections and of other malariological indices in an area of high endemicity. Trans R Soc Trop Med Hyg 1999; 93: S1/15-S1/20.

[33] Peyerl-Hoffmann G, Jelinek T, Kilian A, et al. Genetic diversity of Plasmodium falciparum and its relationship to parasite density in an area with different malaria endemicities in West Uganda. Trop Med Int Health 2001; 6: 607-13.

(C) Sumari et al.; Licensee Bentham Open.

This is an open access article licensed under the terms of the Creative Commons Attribution Non-Commercial License (http://creativecommons.org/ licenses/ by-nc/3.0/), which permits unrestricted, non-commercial use, distribution and reproduction in any medium, provided the work is properly cited. 